\title{
Philosophiques
}

Denis Fisette (dir.), Husserl's Logical Investigations

reconsidered, Dordrecht, Boston, Londres, Kluwer, coll. "Contributions to Phenomenology", 2003, 252 pages.

\section{Pierre-Jean Renaudie}

Volume 33, numéro 2, automne 2006

URI : https://id.erudit.org/iderudit/013901ar

DOI : https://doi.org/10.7202/013901ar

Aller au sommaire du numéro

Éditeur(s)

Société de philosophie du Québec

ISSN

0316-2923 (imprimé)

1492-1391 (numérique)

Découvrir la revue

Citer ce compte rendu

Renaudie, P.-J. (2006). Compte rendu de [Denis Fisette (dir.), Husserl's Logical

Investigations reconsidered, Dordrecht, Boston, Londres, Kluwer, coll.

"Contributions to Phenomenology", 2003, 252 pages.] Philosophiques, 33(2),

533-537. https://doi.org/10.7202/013901ar d'utilisation que vous pouvez consulter en ligne.

https://apropos.erudit.org/fr/usagers/politique-dutilisation/ 
Denis Fisette (dir.), Husserl's Logical Investigations reconsidered, Dordrecht, Boston, Londres, Kluwer, coll. «Contributions to Phenomenology», 2003, 252 pages.

À l'occasion du centenaire de la publication des Recherches logiques, œuvre monumentale dans laquelle la phénoménologie a accompli sa première «percée » si l'on en croit Husserl lui-même, a eu lieu à Montréal un colloque international destiné à revenir sur les origines de la phénoménologie et à en saisir les enjeux dans le cadre de la philosophie contemporaine (notamment en ce qui concerne la logique, l'ontologie, ou encore la philosophie de l'esprit). Les actes de cette rencontre ont servi de point de départ à Denis Fisette pour éditer un recueil d'articles dont l'objectif est de dresser une sorte d'état des lieux des différentes directions offertes un siècle après sa publication par l'œuvre séminale de la phénoménologie husserlienne. Il s'agit à la fois de dégager la signification historique des voies inaugurées par les Recherches et de donner un aperçu des perspectives qu'elles ont inaugurées en laissant ouvertes un certain nombre de directions. Les Recherches logiques donnent une clé intéressante pour aborder la philosophie du $\mathrm{XX}^{\mathrm{e}}$ siècle en se situant tout à la fois dans les débats d'époque auxquels elles espéraient apporter une réponse (psychologisme, fondation des sciences, théorie des multiplicités, méréologie), et à l'origine d'une histoire qui allait durablement séparer philosophie continentale et philosophie analytique, sans pour autant que Husserl s'y engage encore pleinement dans la perspective transcendantale idéaliste qui allait jouer en faveur de leur éloignement réciproque.

Ces différents aspects du projet de relecture des Recherches logiques ont permis à D. Fisette de définir quatre sections thématiques regroupant l'ensemble des contributions de ce recueil. Nous ne présenterons ici que quelques articles significatifs pour en saisir la perspective d'ensemble.

La première section embrasse un dessein général dans l'approche de l'œuvre qu'elle propose, en interrogeant la cohérence et l'unité d'un texte qui apparaît d'abord au lecteur de façon morcelée, découpé en recherches à caractère programmatique et éloignées les unes des autres par la diversité de leurs thèmes. La question de cette unité des Recherches doit ainsi permettre de préciser la portée et le sens du projet philosophique de Husserl. David Woodruff Smith va insister sur la structure systématique de l'œuvre et tenter de reconstruire logiquement l'unité très forte de celle-ci en isolant d'une part les thèses amenées dans chacune des six recherches et dans les Prolégomènes, puis en montrant le lien logique qui les rattache les unes aux autres de façon à constituer une théorie cohérente à partir de laquelle l'ensemble des Recherches logiques trouve un soubassement. L'unité de la théorie est elle-même garantie par la métathéorie mise en place dans les Prolégomènes, laquelle définit les conditions générales de validité des Recherches. Le projet initial de la phénoménologie se noue ainsi autour d'une reconstruction intégrale des différents champs de la philosophie (de la logique à l'épistémologie, en passant 
par une théorie de la signification, des universaux, une ontologie, une grammaire... ) articulés non plus au sein d'un système philosophique mais dans une théorie dont on s'est assuré de la validité formelle, et Smith établit les relations d'interdépendance entre chacune des doctrines.

Denis Fisette prolonge cette perspective en montrant comment le programme d'une théorie de la science présenté par Husserl dans les Prolégomènes lui permet de donner une unité très forte aux deux directions principales dans lesquelles s'engagent les Recherches logiques: la logique pure d'un côté et la phénoménologie de la connaissance de l'autre. L'auteur reconstruit ainsi l'itinéraire intellectuel de Husserl et montre que si la logique pure pose le cadre théorique dans lequel pourront être traitées les tâches fondamentales d'une théorie de la science, cette dernière ne peut s'accomplir que dans une élucidation phénoménologique des principaux concepts qui rendent possible la connaissance objective. D. Fisette fait ainsi apparaître le lien profond qui assure la cohésion des Recherches logiques, mais aussi de l'œuvre de Husserl dans son ensemble puisque ce programme se retrouve, après qu'il eut généralisé l'idée de la logique pure à la totalité des sciences, dans les travaux ultérieurs de Husserl (autour de certains thèmes comme ceux d'une critique de la raison ou d'une philosophie première).

Les deux sections qui suivent sont davantage tournées vers une perspective historique. La seconde section du recueil retrace d'abord les liens étroits et complexes qui rattachent Husserl à son illustre prédécesseur dans la voie d'une "théorie de la science ", à savoir Bolzano. Jan Sebestik dresse un parallèle entre certaines idées centrales des Recherches et la doctrine de la Wissenschaftslehre: en renversant le rapport entre théorie de la connaissance et théorie de la science de façon à subordonner la première à la seconde, Bolzano définissait le cadre général des Prolégomènes. Husserl s'y donne en effet pour tâche de répondre au problème posé par Exner à Bolzano, lequel, ne disposant pas de la méthode phénoménologique, n'avait pas eu les moyens d'y répondre de façon satisfaisante. Il s'agit de comprendre comment il peut y avoir une saisie subjective de significations objectives, si tant est que les secondes n'existent pas (du moins selon Bolzano) alors que la pensée qui s'en empare a bien, quant à elle, une existence psychologique dans l'esprit. L'élucidation phénoménologique de la connaissance aurait ainsi permis à Husserl de traiter cette question tout en suivant Bolzano dans son refus d'adopter la thèse d'une correspondance (pensée sur le mode de la ressemblance) entre les idées ou représentations et leurs objets.

Rolf George reprend dans un article très détaillé cette question du rapport Husserl-Bolzano par le biais du psychologisme, pour donner un éclairage nouveau sur son rôle dans la théorie de la logique de ces deux auteurs. Il est selon lui nécessaire de bien distinguer entre la dénonciation programmatique du rôle joué par la psychologie dans la logique et l'usage de la psychologie dans des contextes logiques. L'auteur indique à cet effet l'usage positif qu'ont fait certains logiciens importants de considérations psychologiques sans pour 
autant tomber dans une forme de psychologisme (notamment en prenant l'exemple de la logique intuitionniste). Mais dans aucun cas une loi psychologique n'a à intervenir pour valider les théorèmes des systèmes logiques en question, malgré la référence à l'esprit humain et aux modes du raisonnement. La psychologie ne justifie donc pas les principes et les lois logiques, même s'il est fait appel à elle dans l'élaboration du système. R. George montre alors que le problème du statut des entités que sont les "propositions en soi » chez Bolzano ne constitue pas une des limites de sa doctrine mais au contraire un remarquable effort pour éviter toute contagion de la logique par une doctrine psychologique. Bolzano propose une solution originale et solide au problème classique du statut des idées en les traitant du seul point de vue de leur fonction logique : il n'est plus question de ressemblance entre nos idées et leurs objets mais de la seule référence des termes de la proposition à leurs objets, sans que ne doive intervenir cette notion de ressemblance. Aussi l'outillage théorique de l'épistémologie bolzanienne lui vient-il de la logique bien davantage que d'une psychologie qui reste chez lui très en arrière-plan (et c'est sans doute pour cette raison que Husserl lui reproche d'être resté en deçà d'une authentique phénoménologie de la connaissance appuyée sur une psychologie descriptive). La position bolzanienne incite donc à ne pas s'arcbouter sur la question du statut ontologique ou de la nature des objets abstraits que sont les propositions : il suffit de dire que notre façon habituelle de parler implique le plus souvent notre croyance qu'il y a des propositions, et que nous sommes tenus de faire comme si les propositions existaient, même s'il n'existe pas de lieu défini pouvant permettre de leur assigner un statut ontologique. Il faut donc interroger les relations entre propositions plutôt que de chercher à atteindre leur essence.

La troisième section se penche sur les liens qui rattachent la pensée des Recherches à l'école brentanienne. La critique adressée par Husserl à Brentano et à sa compréhension des attitudes propositionnelles s'applique selon Richard Cobb-Stevens aussi bien à Frege, qui conçoit le jugement comme une prise de position relative à un contenu propositionnel. C'est sur ce point que va porter la critique de Husserl dans la $5^{\mathrm{e}}$ Recherche, qui rejette l'idée brentanienne d'un contenu de représentation neutre sur lequel porterait ensuite l'affirmation ou le rejet judicatif. Cette critique se précise encore dans Logique formelle et Logique transcendantale, où Husserl critique la conception moderne selon laquelle le contenu propositionnel ferait office d'entité intermédiaire assurant la liaison entre l'acte du discours et l'objet intentionnel auquel il réfère. Juger ne revient pas à combiner des signes mais à agir directement sur le monde en articulant des choses et des situations. Il faut ainsi penser une continuité entre les formes perceptives et les formes syntaxiques, ou entre la sensibilité et la syntaxe propre au jugement. En insistant sur la dimension antéprédicative de l'expérience, Husserl aurait en quelque sorte actualisé la théorie aristotélicienne du jugement. 
La dernière section adopte une approche plus technique : elle examine les enjeux de l'ontologie husserlienne pour sa pensée ultérieure et relativement à des perspectives plus contemporaines, notamment en resserrant le champ d'investigation autour de la théorie du tout et des parties de la $3^{\mathrm{e}}$ Recherche. Cette méréologie nous donne selon Dallas Willard une clé essentielle pour comprendre le type très original de réalisme qui constitue l'arrière-plan des Recherches: la distinction entre moment dépendant et partie indépendante d'un tout fournit à Husserl l'outil théorique lui permettant de déterminer les lois phénoménologiques fondamentales. En effet, si toute entité relève de la distinction entre tout, parties, moments..., "l'esprit lui-même n'est pas une exception mais un autre cas relevant de ces distinctions ontologiques générales ", et c'est là un point essentiel pour comprendre l'élucidation husserlienne de la possibilité de la connaissance. Tout acte intentionnel peut alors être conçu comme un tout dont la qualité et la matière seront les parties, et Willard explique de cette façon que l'objet est bien un moment de l'acte intentionnel qui le vise sans en être pour autant une partie réelle. Il s'agit là selon lui d'un réalisme d'un type nouveau, puisque la conscience ne transcende pas le champ de la connaissance et de ses objets (avec leur structuration catégoriale) ; elle n'est en aucun cas productrice d'un monde catégorialement structuré qui cesserait d'exister en son absence, et il faut bien plutôt dire qu'elle appartient pleinement à ce monde et se plie à la catégorialité qui le constitue. Le recours à la méréologie pour comprendre le rapport de la conscience au monde permet ainsi d'éviter les différentes impasses dans lesquelles se sont engagées les formes successives de réalisme qui jalonnent la philosophie de l'esprit contemporaine.

Partant de la question de la compatibilité entre les déterminations réelles sur lesquelles reposent les lois causales et les déterminations idéales qui structurent les contenus et les lois de la pensée, James Mensch retrace le cheminement qui conduit Husserl des Recherches Logiques jusqu'au texte tardif, l'Origine de la géométrie. La thématique de l'intersubjectivité apporte alors une réponse à une question que se posait déjà l'auteur en 1900, mais de façon unilatérale. Quoique la lettre du texte n'aille jamais ouvertement dans ce sens, Mensch prend pour argument la fonction référentielle de la signification dans les Recherches afin de suggérer l'idée selon laquelle le fonctionnement de l'intentionalité signitive y suppose déjà le niveau de l'intersubjectivité, au sens d'une activité linguistique commune garantissant une certaine stabilité de la référence et fondant l'idéalité des significations. L'intentionalité présuppose le contexte d'un ensemble de locuteurs s'adressant les uns aux autres dans une même langue, contexte nécessaire à la constitution de tout sens identique répondant à une pluralité d'actes d'identification. Le type de détermination exercé sur notre pensée par ces idéalités est alors de nature intersubjective, dans la mesure où elle est induite par la communauté linguistique à laquelle nous appartenons. 
Les thèmes abordés par les différentes études de ce recueil étant très larges et variés, on pourra au final regretter le manque d'unité de l'ensemble et l'écart parfois important qui sépare les études générales, permettant de brosser un tableau clair et intéressant du contexte et des problématiques à l'arrière-fond des Recherches logiques, des articles d'une très grande technicité qui peuvent avoir tendance à détourner l'œuvre de son projet initial. Le recueil se situe dans une certaine ambivalence, entre l'attention accordée aux conditions historiques de production de l'œuvre et la volonté marquée d'en actualiser certains de ses thèmes, ces deux directions ne parvenant pas toujours à s'harmoniser de façon heureuse. Sans doute rencontre-t-on ici deux façons assez divergentes de lire le texte des Recherches. Il est de plus dommage que n'aient pas été séparées les études qui examinent, de façon souvent très éclairante, certaines questions internes aux Recherches (comme le problème de leur unité, du statut philosophique qu'il faut accorder aux thèses qu'elles défendent...) et les études qui les rattachent à la suite de l'œuvre husserlienne, donnant ainsi un éclairage rétrospectif non dénué d'intérêt mais dont on aurait pu attendre qu'il interroge précisément l'écart séparant ces deux moments de l'œuvre. Cependant, une fois ces réserves formulées, il faut souligner l'intérêt du bilan apporté par ce recueil qui fournit un nombre important de matériaux pour comprendre et interpréter le contexte de l'œuvre (lequel nous apparaît aujourd'hui beaucoup plus en détail) et s'efforce en même temps de donner, cent ans après sa parution, une vue d'ensemble des possibilités ouvertes par un texte dont l'originalité et la force sont bien mises en valeur. D'un côté comme de l'autre, les Recherches logiques apparaissent au terme de cet ouvrage collectif comme un formidable champ d'étude dont les potentialités sont encore loin d'avoir été épuisées.

PIERRE-JEAN RENAUDIE

Université Paris IV-Sorbonne

Bertrand Russell, L'art de philosopher, trad. de l'anglais par Michel Parmentier, Québec, Les Presses de l'Université Laval, coll. «Zêtêsis », 2005, 95 pages.

Ce livre destiné aux philosophes débutants est constitué de trois essais rédigés au début des années 1940, qui furent publiés originalement sous le titre The Art of Philosophizing and Other Essays. L'auteur nous fait part d'abord de sa vision de ce que devrait être le cursus philosophique pour ensuite introduire le lecteur à deux disciplines auxquelles il a consacré une grande partie de ses recherches, la logique et les mathématiques, le tout dans le style vivant et accessible que l'on connaît à ce pionnier de la philosophie exacte, très bien rendu par la traduction de Michel Parmentier.

Le premier chapitre explique que le travail du philosophe consiste à pratiquer «L'art de la conjecture rationnelle », expression qui a donné son titre 